\title{
Dynamic In Situ Spectroscopic Ellipsometry of the Reaction of Aqueous Iron(II) with 2,2'-Bipyridine in a Thin Nafion Film
}

Nebojša Pantelić, Chamika M. Wansapura, William R. Heineman and Carl J. Seliskar

\section{Supporting Information}

\section{Optical constants of glass materials}

Precise determinations of the optical constants for the two types of glass substrates used were performed by data acquisition at several angles followed by transmittance measurements at normal incidence. Transmittance values allowed us to determine the extinction coefficients for glass substrates below $450 \mathrm{~nm}$ by simultaneously fitting ellipsometric data using the Cauchy equation (sensitive to $\mathrm{n}$ ) and transmission data (sensitive to $\mathrm{k}$ ) using the Urbach equation:

$$
k(E)=A_{k} \exp ^{B_{k}\left(E-E_{b}\right)}
$$

where $\mathrm{k}(\mathrm{E})$ is energy dependent extinction coefficient, and $\mathrm{A}_{\mathrm{k}}, \mathrm{B}_{\mathrm{k}}$, and $\mathrm{E}_{\mathrm{b}}$ are the fitting

parameters (amplitude, exponential factor, and band edge transition energy, respectively). ${ }^{13}$ The difference between in situ and ex situ measurements was found to be negligibly small indicating that the cell-mounted glass substrates were essentially stress free and not birefringent.

\section{Optical constants of liquids}

The optical constants of low concentration solutions prepared in water as a solvent might be exchanged in the model for the constant of water in many practical cases. However, we found this assumption to result in relatively large errors (approximately one standard deviation) even if our studies involved very low concentration solutions. This influence was the most pronounced 
in the case of bpy solutions. Indeed, the strong absorption of bpy (text Figure 2) in the near-UV region (maximum at $298 \mathrm{~nm}$ ) significantly increased the refractive index in the entire UV-VIS wavelength region in accordance with anomalous dispersion. ${ }^{28}$

We used a previously characterized planar SF11 glass substrate for measurements on liquids using a similar experimental configuration to that of Tiwald et al. ${ }^{15}$ A complication in their work arose from the large systematic errors that were observed as an offset in the indices and in addition to the relatively high noise of their measurements. Our measurements performed in the VIS and near-IR regions were characterized by lower noise. A somewhat higher but yet small index offset (caused by systematic errors) between experimental and generated data was observed at the extremes of the wavelength region $(\lambda \sim 400$ and $1000 \mathrm{~nm})$. The reason for this probably lies in the assumption that $\mathrm{k}(\lambda)=0$ for these liquids. Water optical constants were determined and compared very favorably with Palik's data ${ }^{16}$ as shown in Table $1 \mathrm{~S}$. In addition, the validities of constants in Table $1 \mathrm{~S}$ have been tested and confirmed in the dynamic measurements stages of the experiments.

Table 1S. Cauchy coefficients for non-absorbing, $\mathrm{k}(\lambda)=0$, media.

\begin{tabular}{lllll}
\hline & $\mathrm{A}$ & $\mathrm{B}$ & $\mathrm{C}$ & $\mathrm{n}_{550}$ \\
\hline Water (from Palik) & 1.315 & 0.007 & 0 & 1.338 \\
Water & 1.321 & 0.004 & 0 & 1.334 \\
$0.1 \mathrm{M} \mathrm{NaCl}$ & 1.322 & 0.004 & 0 & 1.335 \\
$3 \times 10^{-3} \mathrm{M} \mathrm{bpy}^{\text {a }}$ & 1.324 & 0.004 & 0 & 1.337 \\
$1 \times 10^{-5} \mathrm{M} \mathrm{Fe}^{2+\mathrm{a}}$ & 1.323 & 0.004 & 0 & 1.336 \\
SF11 glass & 1.742 & 0.015 & 0 & 1.792 \\
$1737 \mathrm{~F}$ glass & 1.502 & 0.005 & 0 & 1.518
\end{tabular}

a. In $0.1 \mathrm{M} \mathrm{NaCl}$ solution 
Optical modeling when dielectric function (refractive index) is complex value

The oscillators are mathematical functions corresponding to optical absorbances that are parameterized with one or more variables and used for the interpretation of optical measurements of many thin-film systems ${ }^{28,30}$ (here, the Nafion-Fe(bpy) ${ }_{3}{ }^{2+}$ system). We used four Tauc-Lorentz oscillators for global modeling derived by multiplying the previously described Tauc and Lorentz oscillators:

$$
\begin{array}{rlrl}
\varepsilon_{2}(E) & =\left[\frac{A E_{0} C\left(E-E_{g}\right)^{2}}{\left(E^{2}-E_{0}^{2}\right)^{2}+C^{2} E^{2}} \frac{1}{E}\right], & & E \succ E_{g} \\
& =0 & , E \leq E_{q}
\end{array}
$$

where $\varepsilon_{2}(E)$ is energy dependent complex dielectric function, $E_{0}$ is oscillator position in the spectrum, $\mathrm{A}$ is amplitude of the oscillator (strength), $\mathrm{C}$ is a band broadening term, and $\mathrm{E}_{\mathrm{g}}$ is the band-gap energy. ${ }^{30}$ The real part of the dielectric function, $\varepsilon_{1}(\mathrm{E})$, is calculated using the Kramers-Kroning relation (vide infra), whereas corresponding $\mathrm{n}$ and $\mathrm{k}$ values using the following equations:

$$
n=\sqrt{\frac{1}{2}\left[\left(\varepsilon_{1}^{2}+\varepsilon_{2}^{2}\right)^{1 / 2}+\varepsilon_{1}\right]}, k=\sqrt{\frac{1}{2}\left[\left(\varepsilon_{1}^{2}+\varepsilon_{2}^{2}\right)^{1 / 2}-\varepsilon_{1}\right]}
$$

The magnitudes (relative to the pole oscillator), position in the spectrum, relative broadening, and the band gap energy of these oscillators are given in Table 2S. This number of oscillators seems to be the minimum necessary for adequate modeling of the $\mathrm{Fe}(\mathrm{bpy})_{3}{ }^{2+}$ absorption spectrum. The simplifying assumption was made that the extinction coefficient wavelength profile in the film essentially was the same as the absorbance of the same complex in solution. The four oscillators were added together and then multiplied by a single computer-fitting parameter. Only the absolute magnitude of this fitting parameter was allowed to vary during the 
experimental data fitting procedure. A pole oscillator with relatively high magnitude at $9.71 \mathrm{eV}$ $(128 \mathrm{~nm})$ was needed to quantitatively describe the film refractive index. Addition of the refractive index offset as a fitting parameter made it possible to describe the refractive index dynamics during $\mathrm{Fe}^{2+}$ partitioning into the Nafion film. To summarize, three experimental data fitting parameters were employed: thickness, extinction coefficient fitting factor, and the refractive index offset.

Fitting three unknown parameters with two measured values, $\Psi$ and $\Delta$, carries with it the possibility of ending up with results that lack uniqueness. However, the uniqueness of the results can be fulfilled by using the self-consistency of the Tauc-Lorentz oscillators (via the KramersKroning relationship, equation A4) with the linked refractive index and extinction coefficient: ${ }^{13,31}$

$$
n(E)-1=\frac{2}{\pi} P \int_{0}^{\infty} \frac{E^{\prime} k\left(E^{\prime}\right)}{E^{\prime 2}-E^{2}} d E
$$

where $\mathrm{E}$ is the energy corresponding to the wavelength of light used and $\mathrm{P}$ denotes that only the principal part of the integral is taken in order to avoid the singularity at E'=E. In other words, the farther away from an absorbance band, the smaller the influence of the extinction coefficient on the refractive index. As a result, the data fitting procedure with three unknowns was reduced to two due to oscillator self-consistency. In turn, the demand for uniqueness of the computer fitting was fulfilled.

The transparent region above $700 \mathrm{~nm}$ in the absorbance spectrum of Fe(bpy) ${ }_{3}{ }^{2+}$ (text Figure 2 , inset) is characterized by $\mathrm{k}(\lambda) \cong 0$ and is very useful to test our results. Only two parameters have to be fitted here, namely, thickness and refractive index offset. Subsequently, 
these results can be compared with those obtained by fitting the entire wavelength region with three unknowns.

Table 2S. Tauc-Lorentz and pole oscillator parameters for $\mathrm{Fe}(\mathrm{bpy})_{3}{ }^{2+}$.

\begin{tabular}{lllll}
\hline & $\mathrm{A}$ & $\mathrm{E}_{0}(\mathrm{eV})$ & $\mathrm{C}(\mathrm{eV})$ & $\mathrm{Eg}(\mathrm{eV})$ \\
\hline Tauc-Lorentz I & 0.24 & 2.32 & 0.92 & 1.95 \\
Tauc-Lorentz II & 0.03 & 2.55 & 1 & 1.70 \\
Tauc-Lorentz III & 0.15 & 2.96 & 0.43 & 2.88 \\
Tauc-Lorentz IV & 0.16 & 3.13 & 0.75 & 2.88 \\
pole & 84.71 & 9.71 & ---- & ----
\end{tabular}

